\title{
IMPLEMENTATION AND DEMONSTRATION OF LI-FI TECHNOLOGY
}

\author{
Abhishek Kurup ${ }^{1}$, Vipin Tiwari ${ }^{2}$, Selvanathiya ${ }^{3}$ \\ ${ }^{1}$ Student, B.Tech, E.C.E, Veltech University, Avadi, Chennai, Tamil Nadu \\ ${ }^{2}$ Student, B.Tech, E.C.E, Veltech University, Avadi, Chennai, Tamil Nadu \\ ${ }^{3}$ Assistant Professor, E.C.E, Veltech University, Avadi, Chennai, Tamil Nadu
}

\begin{abstract}
Li-Fi is a wireless communication system in which light is used as a carrier signal instead of traditional radio frequency as in Wi-Fi. Li-Fi is a technology that uses light emitting diodes to transmit data wirelessly. Li-Fi is a form of Visible Light Communication (VLC). $V L C$ uses rapid pulses of light to transmit information wirelessly that cannot be detected by the human eye. This paper demonstrates the working of Li-Fi by simulating a simple circuit which gave us the required output.
\end{abstract}

Li-Fi technology was first demonstrated by Harald Hass, a German Physicist from the University of Edinburgh

Keywords-Li-Fi, VLC, Optical Communication, Wireless Communication, LED, Visible Light Spectrum. - ***

\section{INTRODUCTION}

Over the past few years there has been a rapid growth in the utilization of the RF region of the electromagnetic spectrum. This is because of the huge growth in the number of mobile phones subscriptions in recent times. This has been causing a rapid reduction in free spectrum for future devices. Lightfidelity ( $\mathrm{Li}-\mathrm{Fi}$ ) operates in the visible light spectrum of the electromagnetic spectrum i.e. it uses visible light as a medium of transmission rather than the traditional radio waves.

The figure below shows a brief idea of the functioning of the Li-Fi system.

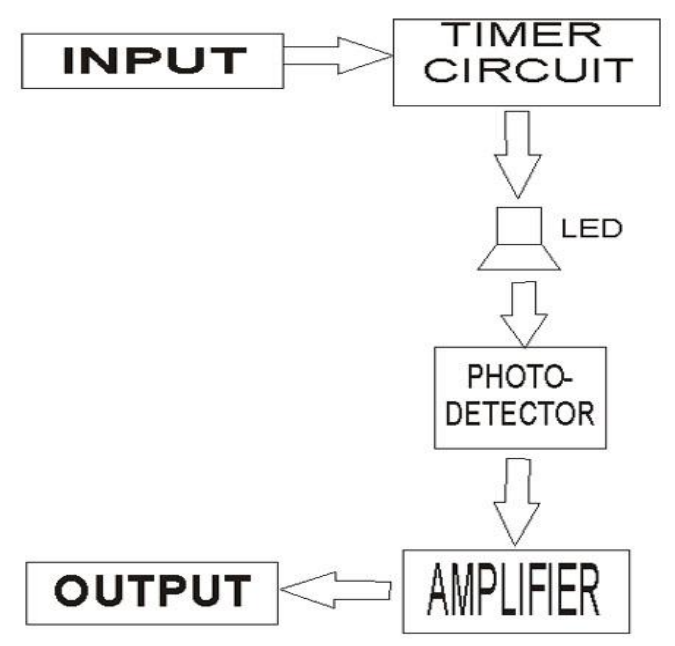

BASIC LI-FI SYSTEM BLOCK DIAGRAM

\section{WORKING}

\subsection{Introduction}

The Li-Fi system consists of mainly two parts, the transmitter and the receiver. The transmitter part modulates the input signal with the required time period and transmits the data in the form of 1's and 0's using a LED bulb. These 1's and 0's are nothing but the flashes of the bulb. The receiver part catches these flashes using a photodiode and amplifies the signal and presents the output.

\subsection{Transmitter}

As per the given diagram, the transmitter section consists of the input, a timer circuit, and a LED bulb. The input can be any type of data that you wish to transmit, for example voice, text etc. The timer circuit is used to provide the required time intervals between each bit. These bits i.e. 1's and 0's are transmitted in the form of flashes of the LED bulb.

\subsection{Receiver}

The flashes of the bulb are received by the photodiode. The photodiode then converts the light energy into electrical signals. Next these electrical signals are amplified and the output is presented.

\section{SIMULATION}

The simulation was carried out using NI Multisim 12.0. The circuit consists of a simple 555 timer connected in astable mode, an optocoupler, and an inverting amplifier. The input frequency is set by using a virtual function generator and the output waveforms are viewed using a virtual oscilloscope. 


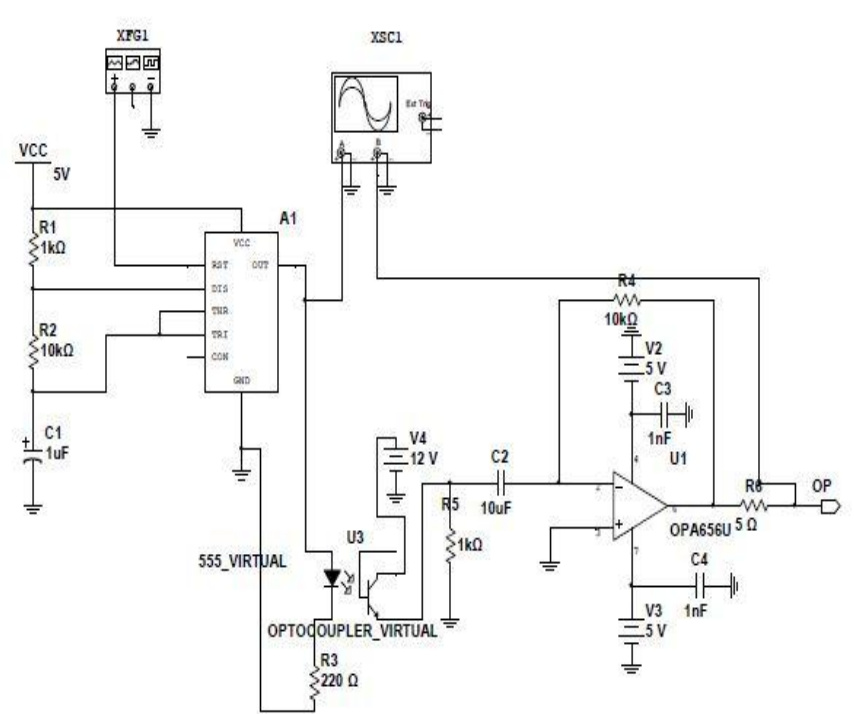

\subsection{Circuit Details}

This simple circuit consists of a timer circuit, an optocoupler and an amplifier circuit. The input frequency is given using a virtual function generator (XFG1). This input is transmitted by converting the signal into square waves using a 555 timer configured in astable mode. These square waves represent the bits 1's and 0's of the data. These electrical signals are then transmitted using an optocoupler. The optocoupler was used because it was not possible to simulate the transmission between an LED and a photodiode.

The output signal from the optocoupler is fed into an inverting amplifier constructed using Opamp OPA656. The input signal from the function generator and the output signal from the amplifier are viewed using the virtual oscilloscope.

The simulation result is shown below.

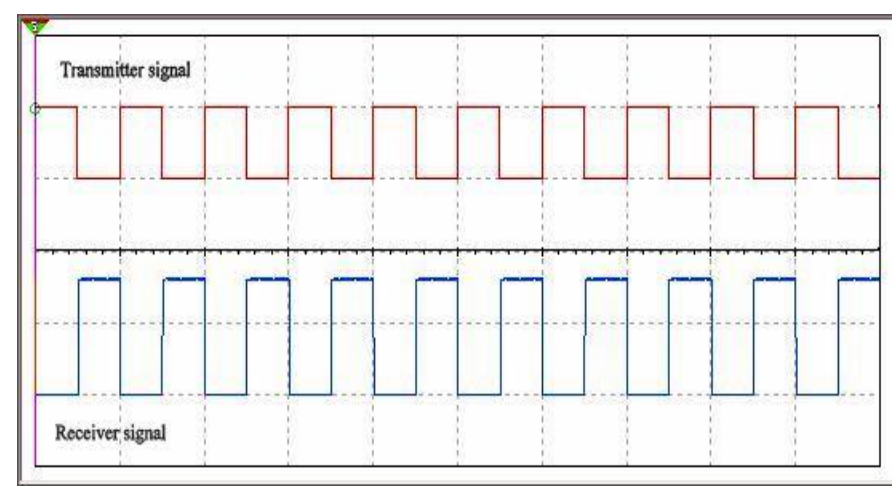

The waveform in the upper section is the input from the function generator and the waveform in the bottom section is the output waveform obtained from the amplifier.

\subsection{Components Details}

555 Timer: The 555 timer IC is an integrated circuit (chip) used in a variety of timer, pulse generation, and oscillator applications. The 555 can be used to provide time delays, as an oscillator, and as a flip-flop element.

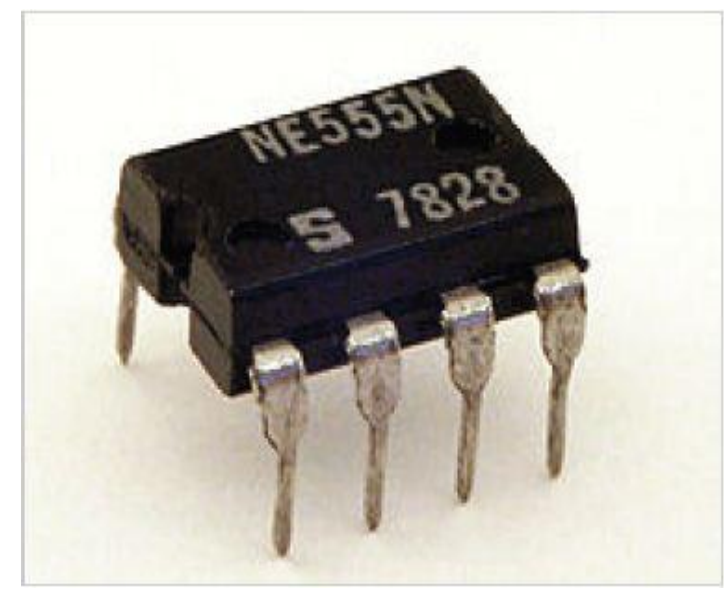

555 TIMER

OPA656: The OPA656 combines a very wideband, unity-gain stable, voltage-feedback op amp with a FET-input stage to offer an ultra-high dynamic-range amplifier for ADC (Analog-to-Digital Converter) buffering and transimpedance applications. Extremely low DC errors give good precision in optical applications. The high unity-gain stable bandwidth and JFET input allows exceptional performance in high-speed, low-noise integrators. The high input impedance and low bias current provided by the FET input is supported by the ultralow $7 \mathrm{nV} / \mathrm{Hz}$ input voltage noise to achieve a very low integrated noise in wideband photodiode transimpedance applications.

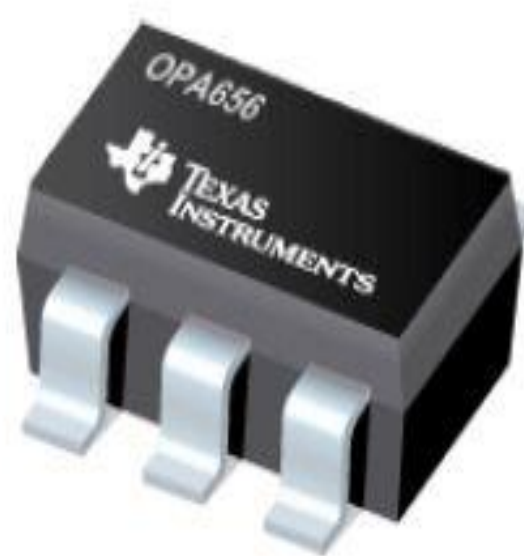

OPA656 


\section{APPLICATIONS}

Visible light communication can prove to be very useful in areas where radio signals cannot be used due to its interference with other appliances. These areas may include hospitals, mines, oil rigs, planes etc.

Li-Fi can also be called as a form of green technology. It is said so because there won't be any harm to animals, birds as well as humans from any kind of radiation.

Underwater communication will be possible between deep sea divers and explorers.

There are many more applications where we can switch from $\mathrm{RF}$ to $\mathrm{Li}-\mathrm{Fi}$ for easier communication.

\section{ADVANTAGES}

High Security: Light cannot pass through walls thus it provides a secure connection to only the users in the particular room.

High Data Transfer Rate: Data is transferred at the speed of light. Thus it can give data transfer speed up to $100 \mathrm{Gbps}$.

Reliable and Safe: Visible light is harmless to every living being thus it does not cause any hazards such as that from RF.

Very less infrastructure cost: Nowadays everyone uses LED bulbs for lighting purpose as a result the infrastructure is already present.

\section{CONCLUSIONS}

From the simulation we can see that it is possible to transmit data using visible light as a medium. Also using the same basic circuit with a few modifications such as usage of DSP and DIP it will also be possible to transmit higher quality of data such as videos and sound apart from just text or simple bits. This type of communication will also reduce the risks off radiation hazards and can be used almost anywhere even at places where electronic devices are banned due to the fear of radiation.

Apart from just transmission of data this technology can also be used to replace the existing $\mathrm{Wi}-\mathrm{Fi}$ system to connect to the internet.

\section{REFERENCES}

[1] M. Thanigavel, "Li-Fi Technology in Wireless Communication," International Journal of Engineering Research \& Technology (IJERT) Vol. 2 Issue 10, October -2013

[2] Megha Goyal, Dimple Saproo, Asha Bhagashra, "New Epoch of Wireless Communication: Light Fidelity", International Journal of Innovative Research in Computer and Communication Engineering Vol. 1, Issue 2, April 2013
[3] http://en.wikipedia.org/wiki/Li-Fi

[4] www.lificonsortium.org/

[5] Thilo Fath, Christoph Heller, and Harald Haas, Member, IEEE,"Optical Wireless Transmitter Employing Discrete Power Level Stepping”, JOURNAL OF LIGHTWAVE TECHNOLOGY, VOL. 31, NO. 11, JUNE 1, 2013.

[6] Harald Haas, 'Wireless data from every light bulb', TED Global, Edinburgh, July 2011.

[7] en.wikipedia.org/wiki/visible_light_communication.

[8] Hany Elgala, Raed Mesleh and Harald Haas, "Indoor Broadcasting via White LEDs and OFDM". 